\title{
Ice-Water Hand Immersion Causes a Reflex Decrease in Skin Temperature in the Contralateral Hand
}

\author{
Youzou ISII ${ }^{1,2}$, Kanji MATSUKAWA ${ }^{1}$, Hirotsugu TSUCHIMOCHI ${ }^{1}$, and \\ Tomoko NAKAMOTO ${ }^{1}$ \\ 1Department of Physiology, Graduate School of Health Sciences, Hiroshima University, Hiroshima, 734-8551 Japan; and \\ ${ }^{2}$ Department of Clinical Engineering, Faculty of Health Sciences, Hiroshima International University, Higashi-Hiroshima, Hiroshima, \\ 724-0695 Japan
}

\begin{abstract}
Cutaneous receptors stimulated by ice-water immersion of one hand will increase sympathetic nerve activity to the palm skin in the nonimmersed contralateral hand and reduce blood flow, reflecting on a decrease in skin surface temperature under a constant ambient environment. To test the hypothesis that gender might affect the contralateral vasoconstrictor response, we analyzed the spatiotemporal pattern of palm skin surface temperature during ice-water immersion for 10 min using thermography in eight males and eight females. As soon as the left hand was immersed in ice-water, palm skin temperature in the nonimmersed right hand quickly decreased in all subjects, particularly in the periphery of the digits and palm. The reduction in skin temperature was short-lasting in $63 \%$ of males and $38 \%$ of females, but it lasted throughout immersion in the remaining
\end{abstract}

subjects. The average decrease in palm skin temperature was not significantly different between males and females, though it tended to be greater in males. The mean arterial blood pressure significantly increased and heart rate decreased during immersion in males, whereas no substantial cardiovascular changes were observed in females. Cold sensation was well coincident with the appearance of a reduction in the palm skin temperature. In consideration of all these results, we suggest that cutaneous cold stimuli increased skin sympathetic nerve activity in the nonimmersed hand and reduced skin blood flow. We also contend that gender difference in the contralateral vasoconstrictor response was denied because the time course and magnitude of the decrease in palm skin temperature were not different between males and females.

Key words: skin blood flow, vasoconstriction, thermography, gender difference, feed-forward control.

It is known that an ice-water immersion of one hand or foot induces vasoconstriction and then vasodilatation in the immersed skin area [1-4]. The immersion stimulates not only cutaneous cold-sensitive receptors, but also nociceptors, which in turn evoke an axon reflex and/or a reflex via the central nervous system [5-7]. The reflex adjustment increases skin sympathetic nerve activity to the immersed area in ice-water, which causes a vasoconstriction of cutaneous blood vessels followed by vasodilatation resulting from the axon reflex [8]. On the other hand, cold stimulus increases sympathetic nerve activity in the skin and skeletal muscle in a nonimmersed limb, which in turn induces vasoconstriction and reduces blood flow to the nonimmersed vascular areas [9-12]. The cutaneous vasoconstrictor response is expected to reflect on a decrease in skin surface temperature under a constant ambient environment.

Recently Isii et al. [13] reported that young females had lower fingertip skin temperature than young males when they examined skin temperatures in the hands of more than 1,000 college students using thermography. Core temperature may be lower in females than males. However, if we assume the same core temperature between young males and females, the gender difference in fingertip skin temperature should be explained by a reduction in skin blood flow, which is due to changes in cutaneous vascular conductance and/or perfusion pressure. Young females may have the lower baseline level of skin blood flow to the fingertip in association with higher skin vasoconstrictor activity and/or lower arterial blood pressure. Since the gender difference in fingertip skin temperature becomes more evident in the cold than in a temperate condition [13], this difference may be explained by a sex-related modulation of the thermoregulatory response in skin blood flow during cold exposure as reported previously [14]. To test this hypothesis, we attempted to determine the effect of gender on the contralateral vasoconstrictor response during the ice-water immersion of one

Received on Jul 13, 2007; accepted on Sep 13, 2007; released online on Sep 15, 2007; doi:10.2170/physiolsci.RP007707 Correspondence should be addressed to: Kanji Matsukawa, Department of Physiology, Graduate School of Health Sciences, Hiroshima University, 1-2-3 Kasumi, Minami-ku, Hiroshima, 734-8551 Japan. Tel: +81-82-257-5435, Fax: +81-82-257-5439, E-mail: matsuk@hiroshima-u.ac.jp 
hand by comparing the changes in fingertip skin temperature between young males and females. The spatiotemporal pattern of the cutaneous vasoconstrictor response in the contralateral hand was measured during ice-water immersion by using two-dimensional thermography, which was able to simultaneously monitor different skin surface temperatures in a certain area.

\section{METHODS}

Subjects. Sixteen subjects [ 8 males and 8 females; age $21 \pm 1$ years $($ mean $\pm \mathrm{SE})]$ volunteered to participate in this study. After the purpose and experimental procedures of this study were fully explained to the subjects, a written informed consent was obtained from each one.

Measurements. Each subject sat for $30 \mathrm{~min}$ in an experimental room at an ambient temperature of $26 \pm 2{ }^{\circ} \mathrm{C}$ and a humidity of $47 \pm 11 \%$. The left hand of each was immersed in ice-water for $10 \mathrm{~min}$. A thermistor (Coretemp CTM-205, TERUMO, Tokyo, Japan) was attached to the 2nd digit to record skin temperature. The thermistor probe was sealed and covered with heat insulating material (polystyrene foam, $1 \mathrm{~cm}$ thick) to minimize the direct effect of the temperature of the circumstance. As soon as the ice-water immersion was ended, moisture around the thermistor was wiped away with a towel. To measure the response of skin temperature in the contralateral right hand of each subject during ice-water immersion, we turned up the palm and recorded the changes in skin surface temperature, using thermography (INFRA-EYE 1200A, FUJITSU, Kanagawa, Japan). The thermographic device had a time resolution of 15 frames/s and a temperature resolution of $0.4^{\circ} \mathrm{C}$ at a range of 29 and $38^{\circ} \mathrm{C}$. The image area was $35 \times 28 \mathrm{~cm}$ with $320 \times 240$ pixels; the minimum decomposition distance was $1.1 \mathrm{~mm}$. All re- corded images were saved on a hard disk. The temperature change was analyzed by specially-designed imageprocessing software with Visual Basic 6.0. The hand area was divided into the four parts (palm, wrist, 1st digit, and 2nd to 5th digit), as shown in Fig. 1. The average temperature of each part was automatically computed as the mean value over pixels with more than $29^{\circ} \mathrm{C}$ involved in that part.

A blood pressure cuff (BP-508 Type S, COLIN, Aichi, Japan) was attached around the right upper arm to record blood pressure at 1 min intervals in all males and six females. Mean arterial pressure (MAP) was calculated as [(systolic arterial pressure $+2 \times$ diastolic arterial pressure)/3]. Heart rate (HR) was obtained from an electrocardiogram signal at the same intervals.

Experimental protocols. The left hand of each subject was immersed in an ice-water bath from the 2 nd -5 th digits up to the middle part of the palm. The water was periodically stirred to maintain its temperature at $0^{\circ} \mathrm{C}$. The left 2nd digit skin temperature was recorded every $1 \mathrm{~min}$ throughout the experiment. The thermographic images on the palm side of each right hand were taken every $10 \mathrm{~s}$ during immersion and every 1 min after. Based on the time course of the temperature changes in the left fingertip, we defined the three time periods (Z1, Z2, and Z3) during the ice-water immersion as shown in Fig. 1. Z1 corresponded to $2.0 \mathrm{~min}$ from the start of immersion, at which the left skin temperature decreased the most rapidly; Z2 was at $5.5 \pm 0.4 \mathrm{~min}$ for males and at $6.9 \pm 0.5 \mathrm{~min}$ for females, at which the left skin temperature maximally decreased in individual subjects (to $6.2-14.7^{\circ} \mathrm{C}$ for males and to $1.3-8.3^{\circ} \mathrm{C}$ for females); $\mathrm{Z} 3$ was at the end of icewater immersion (10 $\mathrm{min}$ ), at which time the left skin temperature increased to a plateau level of $12.3-24.4^{\circ} \mathrm{C}$, except for 2 females. The average temperatures in the ipsi-
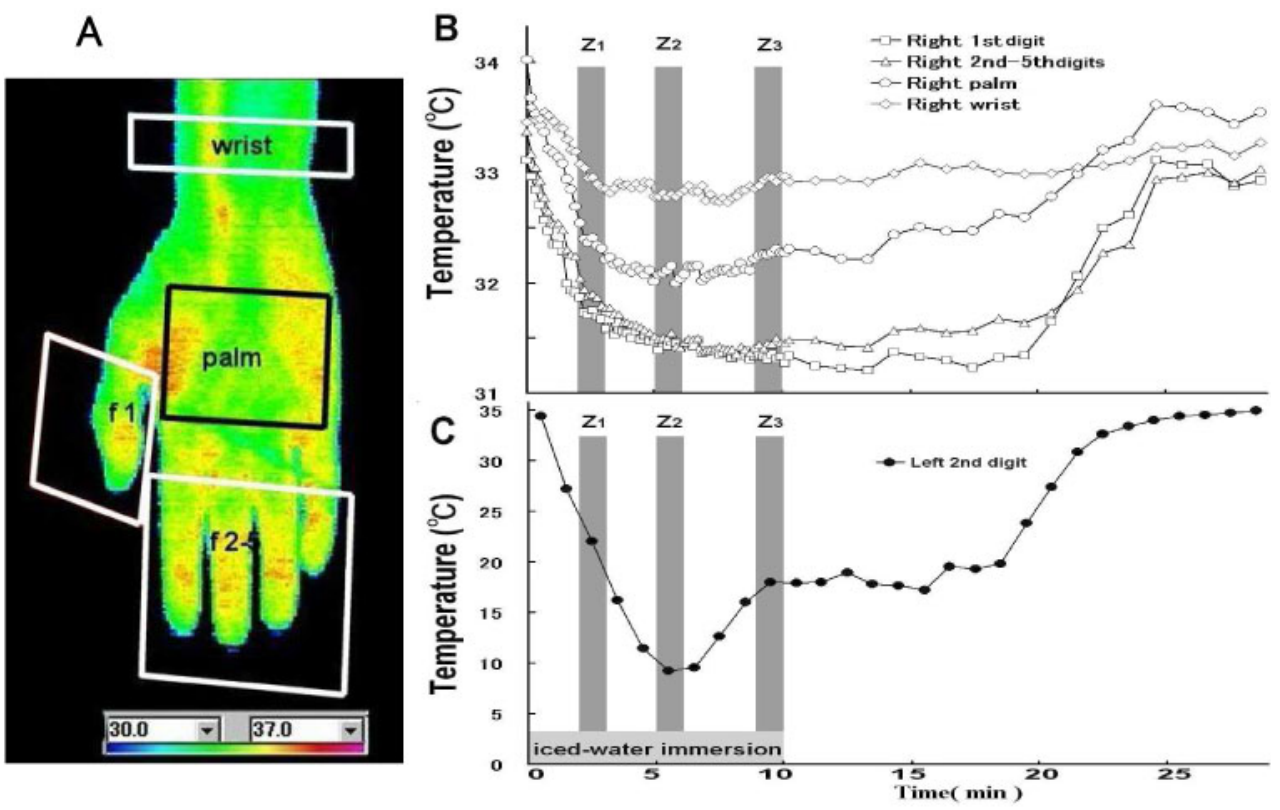

Fig. 1. The changes in skin temperature during ice-water immersion of the left hand for $10 \mathrm{~min}$ in a male. $\mathrm{Z1}$, the period at 2 min during immersion; $Z 2$, the period at which the left fingertip temperature reached the minimum; $Z 3$, the period at the end of immersion (10 min). A: a thermographic image of skin temperature on the palm side of the right hand. $\mathbf{B}$ : The changes in skin temperature on the palm side of the right hand measured with thermography. $\square$, the right 1st digit; $\triangle$, the right 2 nd -5 th digits; $\bigcirc$, the right palm; $\diamond$, the right wrist. C: Skin temperature in the left 2 nd digit measured with a thermistor. 
lateral left 2nd fingertip and the four parts of the contralateral right hand, MAP, and HR were compared among the four periods (before, Z1, Z2, and Z3).

Statistical analysis. The mean values of skin temperature, MAP, and HR were statistically analyzed using a two-way analysis of variance (ANOVA) with repeated measures, which had the two main effects: times (before, Z1, Z2, and Z3) and groups (males and females). When a significant F-value in the main effects was present, a Dunnett post hoc test was performed to see if there was a significant difference between the baseline control and the value at a given time. The level of statistical significance was defined as $P<0.05$. Furthermore, we noted a feeling of coldness and pain during ice-water immersion in individual subjects. All data in the text and figures are expressed as means $\pm \mathrm{SE}$.

\section{RESULTS}

\section{Skin temperature change on the immersed side}

The changes in the left 2nd-fingertip temperature during ice-water immersion in a male are exemplified in Fig. 1. A quick decrease in temperature from the baseline level of $34.9^{\circ} \mathrm{C}$ was observed after ice-water immersion was started. The temperature decreased to the minimum value of $9.2^{\circ} \mathrm{C}$ at $5 \mathrm{~min}$ from the start, i.e., the $\mathrm{Z} 2$ period. Thereafter the fingertip temperature increased and reached a plateau level of $18.4^{\circ} \mathrm{C}$, though ice-water immersion was not ended. Following immersion, the left 2nd-fingertip temperature slowly returned to the baseline level within approximately $25 \mathrm{~min}$.

The time courses of skin temperature in the left 2 nd finger during ice-water immersion are superimposed over all males and females in Fig. 3. The average skin temperatures of the left fingertip before and during ice-water immersion are compared between males and females in Fig. 4. The baseline temperatures of the left fingertips did not differ between the two groups. The left fingertip temperature decreased at the $\mathrm{Z} 1$ and $\mathrm{Z} 2$ periods and thereafter increased to a plateau level in most subjects; the cold-induced vasodilatation appeared in all males and in 6 (out of 8) females (Fig. 3). The left fingertip temperatures at the $\mathrm{Z} 1$ and $\mathrm{Z} 2$ periods were significantly lower in females than in males (Fig. 4).

\section{Skin temperature change on the nonimmersed side}

Skin temperatures in the four parts of the contralateral nonimmersed hand were measured using thermography as exemplified in Figs. 1 and 2. Immediately after the onset of ice-water immersion, skin temperatures in the contralateral hand decreased in all subjects, irrespective of sex, and the decreases in skin temperatures occurred in the peripheries, such as the fingertips (Fig. 2). The time courses of the changes in skin temperatures on the nonimmersed sides are superimposed over all males and females in Fig. 3. There were two distinct types of responses in
A Male

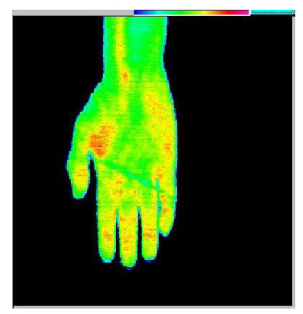

Before

B Female

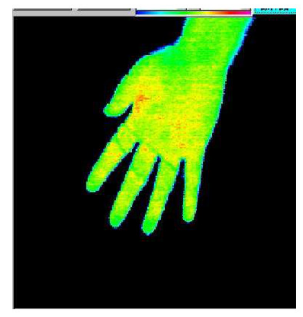

Before

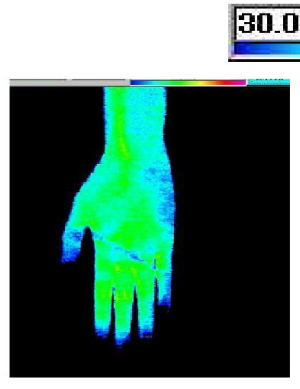

Z1

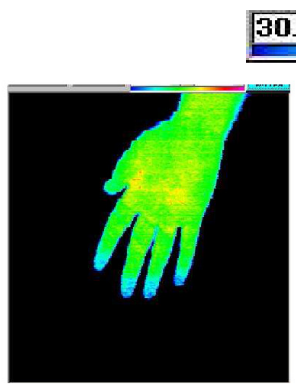

$\mathbf{Z 1}$

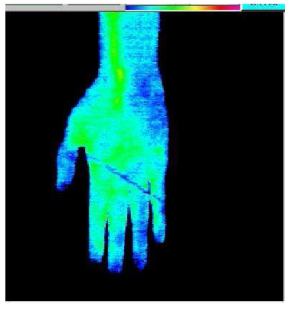

$\mathbf{Z 2}$

30.0

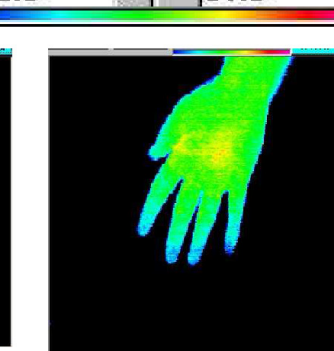

$\mathbf{Z 2}$

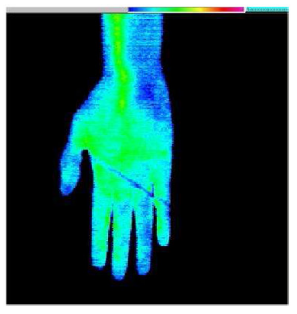

$\mathbf{Z 3}$

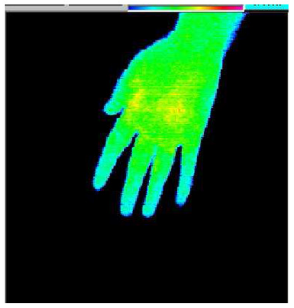

$\mathbf{z 3}$

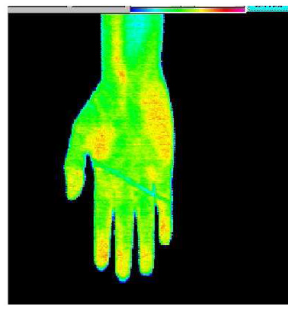

After



After

Fig. 2. The thermographic changes in palm skin temperature of the contralateral right hands before, during (Z1, Z2, and $Z 3)$, and after ice-water immersions of the left hands in a male and a female. 

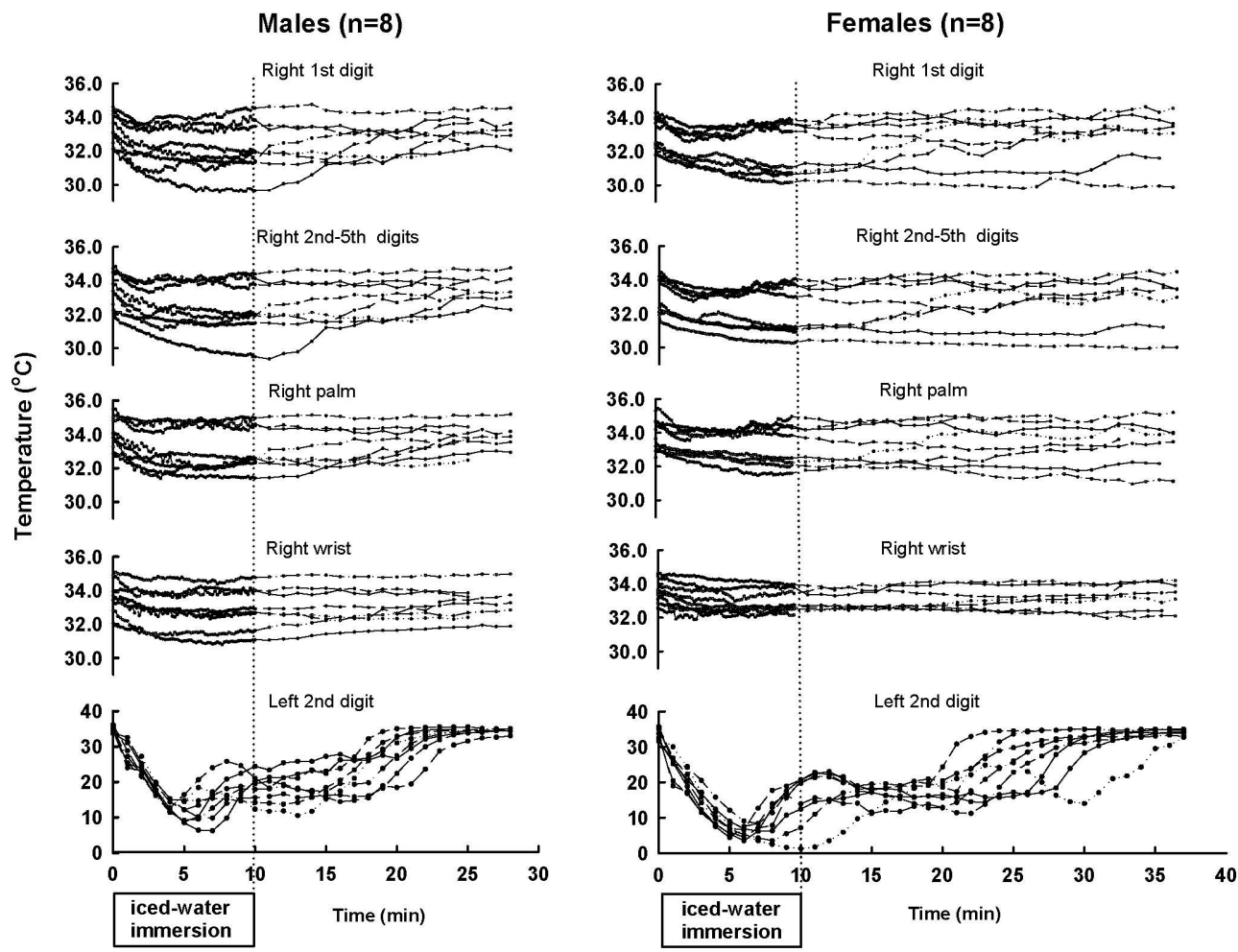

Fig. 3. The time courses of the left fingertip skin temperature and the right palm skin temperatures (four sites) during ice-water immersion. The data are superimposed over 8 males and 8 females.

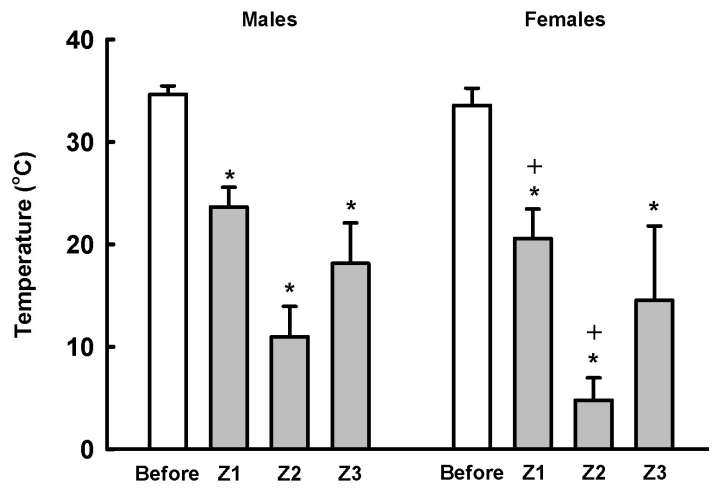

Fig. 4. The average temperature in the left 2 nd fingertip before and during ice-water immersion (Z1, Z2, and Z3) in 8 males and 8 females. The reduction in the left fingertip skin temperature at the $\mathrm{Z} 1$ and $\mathrm{Z} 2$ periods was greater in females than in males. * Significant changes $(P<0.05)$ from the preimmersion control. + Significant difference $(P<0.05)$ between males and females. The data are expressed as means \pm SE.

skin temperatures, i.e., a short-lasting response in 5 of 8 males and 3 of 8 females and a sustained response in the remaining subjects. In the short-lasting type, palm skin temperatures on the nonimmersed sides reached the lowest value prior to the $\mathrm{Z} 2$ period (before the left fingertip temperature reached the minimum) and then gradually returned to the control during the later period of immersion. In the sustained type, the decrease in skin temperature lasted throughout the ice-water immersion (Fig. 3).

It was noted that baseline skin temperatures in females with the sustained response were significantly lower than that in females with the short-lasting response (for example, $32.5 \pm 0.9^{\circ} \mathrm{C}$ vs. $34.1 \pm 0.1^{\circ} \mathrm{C}$ for the $2 \mathrm{nd}-5$ th digits, respectively). In males, baseline skin temperatures with the sustained response also tended to be lower than those with the short-lasting response, though the difference was insignificant.

The average reductions in skin temperatures of the 1st digits, 2nd to 5th digits, palms, and wrists are compared between males and females in Fig. 5. All temperature decreases in the skin areas were significant from the control before immersion. Furthermore, the temperature decrease was greater in the digits and palm than in the wrist in males (Fig. 5). On the other hand, no significant gender differences were observed in any decrease of palm skin temperatures, though the skin temperature reductions tended to be greater in males (Fig. 5). Also, there were no significant relationships in the temperature responses between the immersed and nonimmersed sides, irrespective of a sampling period and gender (Fig. 6), suggesting that the skin temperature responses on the nonimmersed side did not correlate directly to the skin temperature decreases on the immersed side.

\section{The changes in arterial blood pressure and heart rate}

The baseline value of MAP was similar between males and females (95 \pm 4 vs. $86 \pm 6 \mathrm{mmHg}$, respectively); also, the baseline HR was similar between males and females ( $83 \pm 4$ vs. $80 \pm 4$ beats/min, respectively). MAP significantly increased to $112 \pm 3 \mathrm{mmHg}$ at the $\mathrm{Z} 1$ period during ice-water immersion, and HR decreased to $72-73$ beats/ 


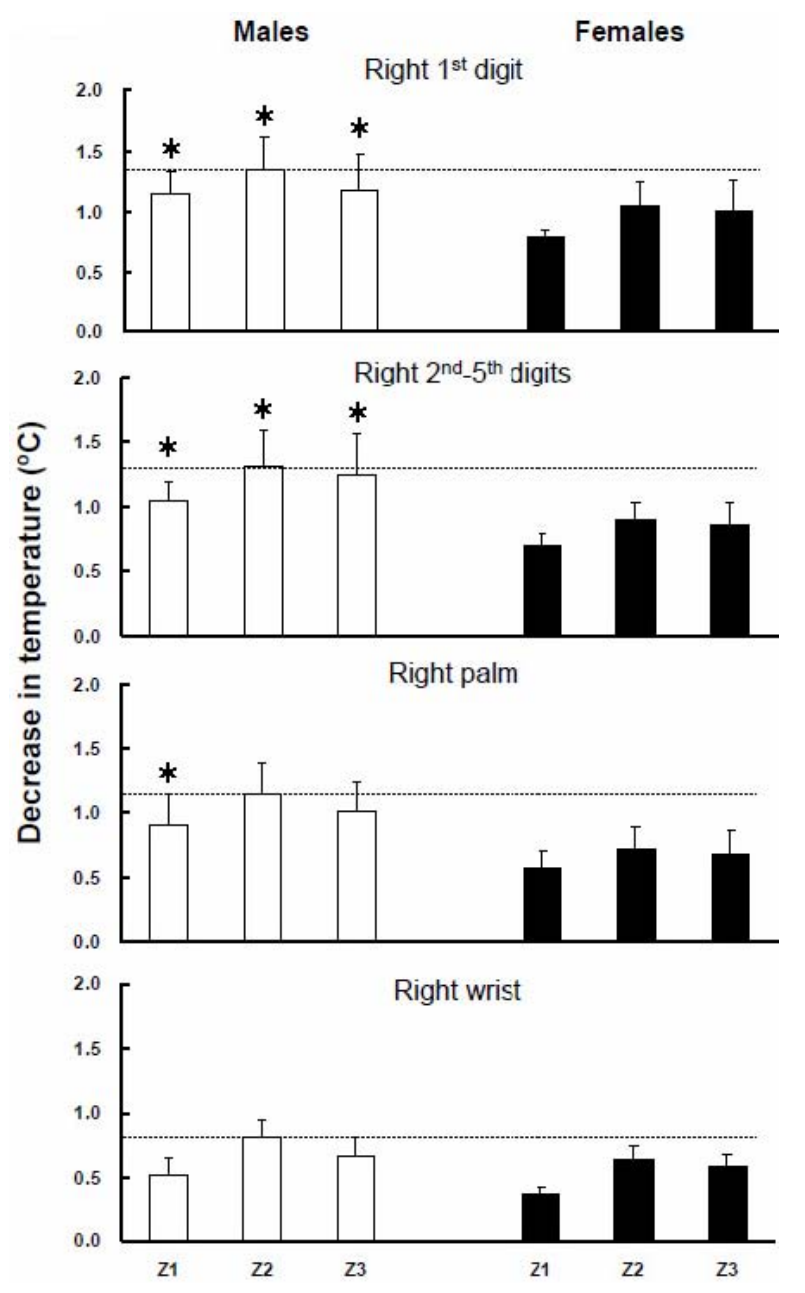

min throughout the immersion in males; whereas they did not change significantly in females. MAP at the Z1 period was significantly higher in males than in females.

\section{Cold and nociceptive sensation}

Immediately after immersion, all subjects felt coldness, but they perceived no noxious stimuli, irrespective of gender. When the left fingertip temperature decreased to nearly $20^{\circ} \mathrm{C}$ at the $\mathrm{Z} 1$ period, sensation ranged from not painful to somewhat painful. It then became intensely painful, like a squeeze, when the left fingertip skin temperature reached near the minimum level around the Z2 period. Thereafter most subjects felt a relief of pain and warmth during the later period of ice-water immersion as the left fingertip temperature increased as a result of vasodilatation, though immersion was not ended.

Fig. 5. The average decrease of skin temperature in the contralateral right hand during ice-water immersion in 8 males and 8 females. The horizontal dotted lines show the levels of the temperature decreases at the Z2 period in males. All decreases of skin temperature were significantly different $(P<0.05)$ from the preimmersion control. The temperature decreases were significantly greater in the periphery of the digits and palms $(P<0.05$ indicated by *) than those in the wrists in males. On the other hand, there were no significant gender differences in the responses of palm skin temperatures in the contralateral hands during immersion. The data are expressed as means \pm SE.
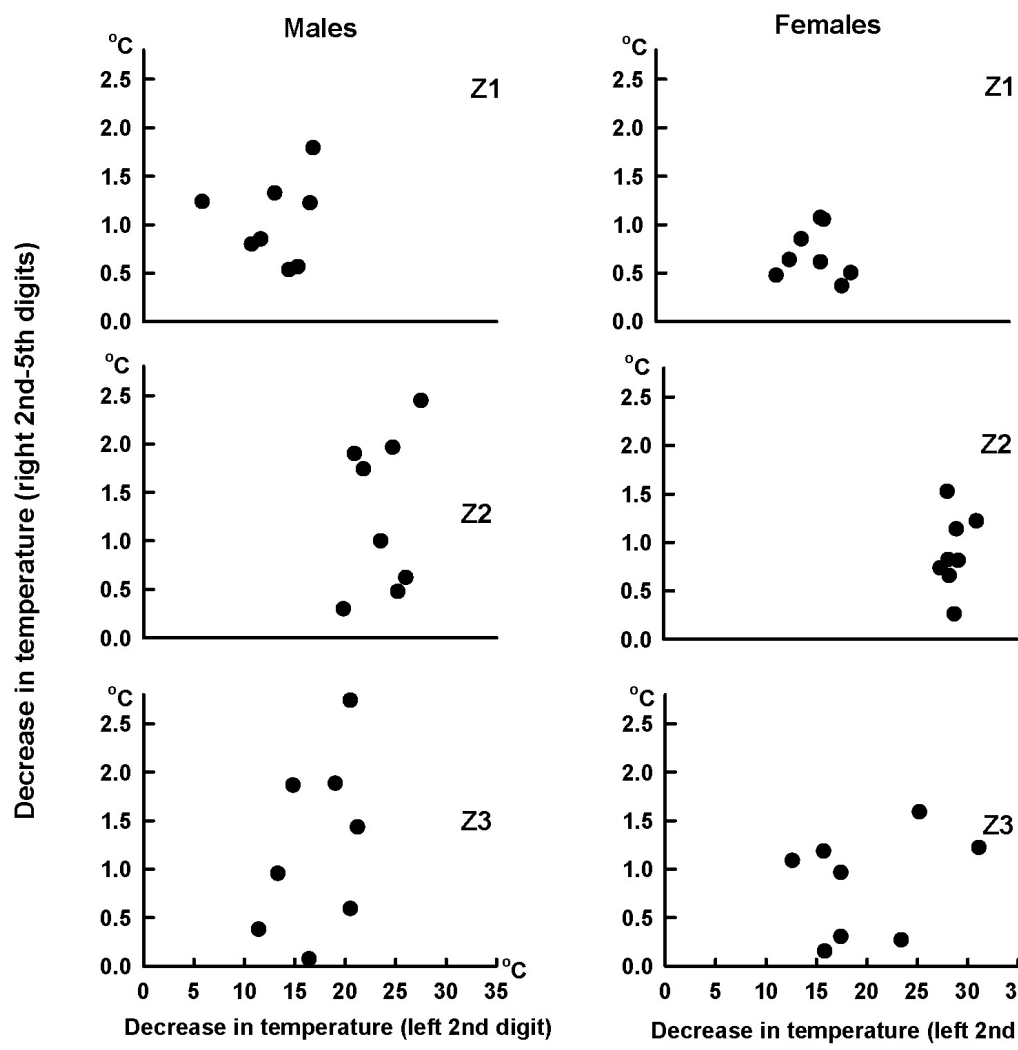

Fig. 6. The relationships in the skin temperature responses between the right 2 nd-5th digits and the left 2nd digit were redrawn from the data shown in Figs. 4 and 5 . There were no significant $(P>0.05)$ relationships between them, irrespective of a sampling period and gender.

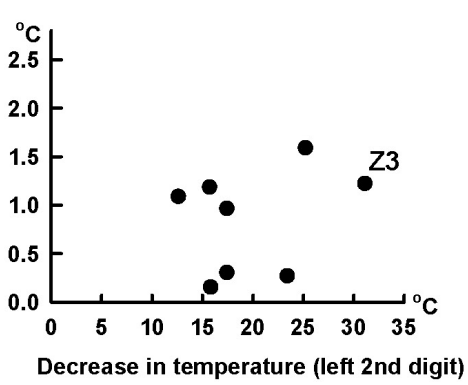




\section{DISCUSSION}

Cutaneous receptors stimulated by ice-water immersion will reduce blood flow to a nonimmersed skin area via increased sympathetic nerve activity, reflecting on a decrease in skin surface temperature under a constant ambient environment. The spatiotemporal pattern of palm skin surface temperatures in the nonimmersed hand during icewater immersion for 10 min was studied using thermography to examine the gender effect on the thermoregulatory response in skin blood flow. We found rapid decreases in palm skin temperatures in the nonimmersed hands, which started immediately after immersion and lasted for several minutes in half of the subjects or were sustained throughout the immersion in the remaining subjects. The decreases in skin temperature were greatest in the peripheries, such as the fingertips. Arteriovenous anastomosis, which richly exists in the fingertips, may be closed by augmented skin sympathetic nerve activity in response to ice-water immersion. Cold sensation was well coincident with the appearance of the reductions in palm skin temperatures, suggesting that a cutaneous cold stimulus will increase skin sympathetic nerve activity to the nonimmersed hand and reduce skin blood flow. However, a gender difference in the contralateral thermoregulatory response was denied because the time course and magnitude of the decreases in palm skin temperatures in the nonimmersed hands were not significantly different between young males and females.

\section{Limitations}

Several substantial limitations regarding the experimental design and data explanation are discussed. First, a sample size of the subjects involved in this study may be insufficient to obtain a definitive conclusion. Second, a female menstrual cycle phase and plasma concentrations of female sexual hormones were not identified in this study, though pain perception to the cold pressor test is known to alter in relation to a menstrual cycle phase $[15,16]$. If the experiments are conducted in a settled menstrual cycle phase, the gender difference about the reflex reduction in the skin temperature of the nonimmersed hand would be established. Third, we measured neither skin blood flow nor sympathetic nerve activity. Although the cutaneous vasoconstrictor response is expected to reflect on a decrease in skin surface temperature under a constant ambient environment, the responses in blood flow and sympathetic nerve activity to a nonimmersed skin area remain to be solved. Fourth, a cuff of an automatic sphygmomanometer was intermittently inflated to measure arterial blood pressure, which might decrease forearm blood flow and affect the measurement of palm skin temperature in the nonimmersed hand. However, this possibility is unlikely because we observed no rapid decrease in skin temperature in synchronization with the cuff inflation (as shown in Fig. 3). Also, when arterial blood pressure was not recorded in some subjects, the responses of skin temperature in the nonimmersed hands were the same as those in subjects with blood pressure measurement. Fifth, a sealing of the thermistor probe on the left fingertip with heat insulating material might affect the time course and magnitude of skin surface temperature. However, such a possibility is unlikely because the time course and magnitude of the left fingertip skin temperature in this study were in good agreement with those of fingertip skin temperatures during cold water immersions in previous studies, in which skin surface temperatures were measured with a thermistor or thermocouple attached to the pad or nail bed of the index or middle finger by a thin layer of adhesive tape $[2,4,12]$.

\section{Cold and nociceptive sensations}

Ice-water immersion stimulates not only cutaneous cold-sensitive receptors, but also nociceptors, which in turn may produce an axon reflex and/or a reflex via the central nervous system. It is meaningful to take into consideration the relationship between thermal and nociceptive sensations and the changes in ipsilateral and contralateral fingertip skin temperatures. Although all subjects felt coldness immediately after immersion, they perceived no noxious stimulus until the left fingertip temperature decreased below $20^{\circ} \mathrm{C}$ after the $\mathrm{Z} 1$ period, in agreement with a previous study [12]. Thus cold sensation was well coincident with the appearance of the reduction in skin temperature of the contralateral fingertips, suggesting that cutaneous cold stimulus will increase skin sympathetic nerve activity to the nonimmersed hand and reduce skin blood flow. It is interesting that when sensation turned painful around the $\mathrm{Z} 2$ period, painful sensation did not always lead to a further decrease in skin temperature on the nonimmersed side.

\section{Changes in fingertip skin temperatures on the immersed side}

Fingertip skin temperature on the immersed side decreased to $5-11^{\circ} \mathrm{C}$ at $6-7 \mathrm{~min}$ after the onset of immersion and then increased to a plateau level, though the immersion was not ended, in agreement with previous studies [1-4]. The reduction in the fingertip skin temperature was significantly greater in females than in males, which may relate to a gender difference in surface area to volume ratio of a hand. A less heat capacity with a smaller hand may cause a greater heat loss from the hand to ice-water. Also, if ice-water immersion evokes vasoconstriction and then vasodilatation in the immersed skin area [2-4], exaggerated vasoconstriction and/or attenuated vasodilatation in the immersed skin area cannot be neglected as another factor to limit blood flow and heat supply in females. 


\section{Change in palm skin temperature on the nonimmersed side}

Afferent input from cutaneous cold-sensitive receptors and/or nociceptors in the immersed hand will be transmitted to the central nervous system, whose autonomic output may elicit the vasoconstrictor response of the contralateral skin blood vessels. Indeed, a rapid increase in skin sympathetic nerve activity in the nonimmersed contralateral hand has been reported [9]. Assuming that afferent input from the cutaneous receptors is in proportion to the reduction in skin temperature in the immersed hand, the contralateral vasoconstrictor response would follow the skin temperature reduction. In fact, the skin temperature response on the nonimmersed side did not match the skin temperature response on the immersed side as follows. First, the time course of the skin temperature change on the nonimmersed side was quite different from that on the immersed side (Fig. 3). Second, there was no significant correlation between the magnitudes of the skin temperature responses on the immersed and nonimmersed sides, irrespective of a sampling period and gender (Fig. 6 ). Based on this evidence, we speculate that input from cutaneous receptors stimulated by ice-water immersion may elicit the spatiotemporally patterned vasoconstrictor response in the nonimmersed hand without direct feedback path, and, in brief, the reflex may be considered as a feed-forward control system.

It is noted that the baseline level of fingertip skin temperature may influence the contralateral reflex response in skin temperature during immersion. When baseline skin temperature was higher than $33^{\circ} \mathrm{C}$, the response in palm skin temperature on the nonimmersed side was short-lasting (Fig. 3). But, when the baseline temperature was lower than $33^{\circ} \mathrm{C}$, the response in palm skin temperature was sustained throughout the immersion. An increase in baseline skin sympathetic nerve activity, associated with a lower baseline skin temperature, may relate to an exaggerated reflex reaction in skin temperature in response to cold stimulus [14]. The effect of a baseline skin temperature on the reflex contralateral response seems independent of gender. However, since baseline skin temperature is higher in males than in females, the short-lasting type of reflex response in palm skin temperature appears more frequently in males.

\section{Effects of gender on the cardiovascular response and the palm skin temperature response on the nonimmersed side}

The effect of gender on the cardiovascular response during ice-water immersion is controversial. McLean et al. [17] reported that males have a greater pressor response to a cold pressor test than females, whereas Jones et al. [18] reported that the cardiovascular and muscle sympathetic nerve responses to a cold pressor test were the same between the two groups. We observed that males, but not females, showed the significant pressor response to ice-water immersion. Based on the present evidence, it is very likely that gender may influence the cardiovascular response during ice-water immersion. Similarly, the response in palm skin temperature on the nonimmersed side tended to be greater in males than in females, despite a smaller reduction in skin temperature on the immersed side (Figs. 3 and 5), though the gender difference in the skin temperature response was insignificant. The reason for controversy among the studies remains to be solved, and a more comprehensive study will be needed to obtain a definitive conclusion about the gender effect on the cardiovascular and thermoregulatory responses to ice-water immersion.

In conclusion, it is very likely that afferent input from cutaneous cold receptors stimulated by ice-water immersion may elicit the spatiotemporally patterned vasoconstrictor response in the nonimmersed hand because the cold sensation was well coincident with the appearance of the reduction in the palm skin temperature. Furthermore, a gender difference in the contralateral vasoconstrictor responses may be denied because the time course and magnitude of the decreases in palm skin temperatures were not different between males and females.

\section{REFERENCES}

1. Lewis T. Observations upon the reaction of the vessels of the human skin cold Heart 1930;15:177-208.

2. Sendowski I, Savourey G, Besnard Y, Bittel J. Cold induced vasodilatation and cardiovascular response in humans during cold water immersion of various upper limb areas. Eur J Appl Physiol 1997;75:471-477.

3. Sendowski I, Savourey G, Launay JC, Besnard Y, Emard JMC, Pequignat JM, Bittel J. Sympathetic stimulation induced by hand cooling after cold-induced vasodilatation in humans. Eur J Appl Physiol 2000;81:303-309.

4. O'brien C, Montain SJ. Hypohydration effect on finger skin temperature and blood flow during cold-water finger immersion. J Appl Physiol 2003;94:598-603.

5. Brain SD, Petty RG, Lewis JD, Williams TJ. Cutaneous blood flow responses in the forearms of Raynaud's patients induced by local cooling and intradermal injection of CGRP and histamine. Br J Clin Pharmacol 1990;30:853-9.

6. Hornyak ME, Naver HK, Rydenhag B, Wallin BG. Sympathetic activity influences the vascular axon reflex in the skin. Acta Physiol Scand 1990;139:77-84.

7. Wallin BG. Neural control of human skin blood flow. J Auton Nerv System 1990;30:185-190.

8. Kunimoto M. Evaluation of the skin sympathetic function by the decrease of skin temperature at the finger tip immersed into cold water (in Japanese). Clin Neurol 1989;29:1004-8.

9. Kunimoto M, Mannen T. The changes of the skin temperature and the skin sympathetic nerve activity recorded by microneurography during cold water immersion of a fingertip (in Japanese). Autonomic Nervous System 1989;26:4854.

10. Victor RG, Leimbach WN Jr., Seals DR, Wallin BD, Mark AL. Effects of the cold pressor test on muscle sympathetic nerve activity in humans. Hypertension 1987;9:429-36.

11. Yamamoto K, Iwase S, Mano T. Responses of muscle sympathetic nervous system activity and cardiac output to the cold pressor test. Jpn J Physiol 1992:42:239-52.

12. Kregel KC, Seals DR, Callister R. Sympathetic nervous system activity during skin cooling in humans: relationship to stimulus intensity and pain sensation. J Physiol 1992;454:359-71.

13. Isii $Y$, Takao F. Examination of fingertip temperature by the thermography measurement seen at the seasonal shift term (in Japanese). Biomed Thermol 
Y. ISIl et al.

2004;23:165-172.

14. Nagashima K, Yoda T, Yagishita T, Taniguchi A, Hosono T, Kanosue K. Thermal regulation and comfort during a mild-cold exposure in young Japanese women complaining of unusual coldness. J Appl Physiol 2002;92:1029-35.

15. Hellstrom B, Lundberg U. Pain perception to the cold pressor test during the menstrual cycle in relation to estrogen levels and a comparison with men. Integr Physiol Behav Sci 2000;35:132-41.

16. Hapidou EG, Catanzaro D. Sensitivity to cold pressor pain in dysmenorrheic and non-dysmenorrheic women as a function of menstrual cycle phase. Pain

1988;34:277-86.

17. McLean JK, Sathasivam P, MacNaughton K, Graham TE. Cardiovascular and norepinephrine responses of men and women to two cold pressor tests. Can J Physiol Pharmacol 1992;70:36-42.

18. Jones PP, Spraul M, Matt KS, Seals DR, Skinner JS, Ravussin E. Gender does not influence sympathetic neural reactivity to stress in healthy humans. Am J Physiol 1996; 270: H350-7. 